\title{
Effect of Perioperative Glucose-Insulin-Potassium Therapy in Patients Undergoing On-Pump Cardiac Surgery: A Meta-Analysis
}

\author{
Qi Li, ${ }^{1,2}$ Jun Yang, ${ }^{1,2}$ Jing Zhang,,3 Chaojun Yang,, 2,3 Zhixin Fan,,2 Ying Yang, ${ }^{1,2}$ Tao Zheng,,$^{1,2}$ Jian Yang ${ }^{1,2}$ \\ ${ }^{1}$ Department of Cardiology, The First College of Clinical Medical Science, China Three Gorges University, Yichang, Hubei, China; \\ ${ }^{2}$ Institute of Evidence-Based and Translational Medicine, China Three Gorges University, Yichang, Hubei, China; ${ }^{3}$ Central Laboratory, \\ Yichang Central People's Hospital, Yichang, Hubei, China
}

\section{ABSTRACT}

Objective: The role of glucose-insulin-potassium (GIK) infusion during cardiac surgery has held interest for so many years without a clear answer. The aim of this meta-analysis was to evaluate the effect of GIK therapy on outcomes in patients undergoing on-pump cardiac surgery.

Methods: A comprehensive online review was performed in The Web of Science, Embase, Medline, PubMed, and The Cochrane Library databases from 2000 to 2019. Eligible studies included randomized controlled trials (RCTs) that compared GIK treatment with placebo or standard care during on-pump cardiac surgery. Risk ratios (RR) were used for binary outcomes and mean difference (MD) was used for continuous variables; both with their $95 \%$ confidence intervals (CI).

Results: A total of 18 RCTs involving 2,131 patients met the inclusion criteria. Compared with the control group, the GIK treatment significantly reduced in-hospital mortality $(\mathrm{RR}=0.56,95 \%$ CI: $0.32-0.97 ; P=.04)$, postoperative myocardial infarctions $(\mathrm{MI})(\mathrm{RR}=0.71,95 \% \mathrm{CI}$ : $0.56-0.91$; $P=.006)$, the use of inotropic support $(\mathrm{RR}=0.53,95 \% \mathrm{CI}$ : $0.45-0.63 ; P<.00001)$, and length of stay in the intensive care unit (ICU) (MD $=-0.33,95 \%$ CI: $-0.52-0.14 ; P=.0007)$. Moreover, GIK treatment seemed to be associated with fewer postoperative atrial fibrillation $(\mathrm{AF})(\mathrm{RR}=0.81,95 \% \mathrm{CI}$ : $0.64-1.03 ; P=.09$ ).

Conclusions: In patients undergoing on-pump cardiac surgery, GIK infusion has a beneficial role in mortality during hospital stay and demonstrates superior efficacy versus standard care for reduction in postoperative MI, AF, ICU length of stay as well as inotropic agent requirements.

\section{INTRODUCTION}

Cardiovascular disease is one of the main causes of morbidity and mortality in the developed world and more than 1.5 million cardiac surgery are per formed each year worldwide

Received September 15, 2019; accepted December 13, 2019.

Correspondence: Fun Yang, MD and fian Yang, MD, Department of Cardiology, The First College of Clinical Medical Sciences, China Three Gorges University, Yiling Road 183, Yichang 443000, Hubei Province, China, 86-18672456622 (e-mail:yangjun@ctgu.edu.cn; yangian@ctgu.edu.cn).
[Benjamin 2018; Laslett 2012]. The use of cardiopulmonary bypass (CPB) is required in many cardiac surgeries. However, it is obvious that on-pump cardiac surgery inevitably causes many kinds of postoperative complications, including atrial fibrillation $(\mathrm{AF})$, myocardial infarction $(\mathrm{MI})$, and infection, which is associated with prolonged intensive care unit (ICU) stays and a patient's long-term prognosis [Giomarelli 2003; Mathew 2004]. Several prophylactic strategies have been developed to reduce these deleterious effects and improve clinical outcomes following cardiac surgical procedures using CPB.

Glucose-insulin-potassium (GIK) commonly has been applied as adjuvant therapy in cardiac surgery, mainly because of its potentially beneficial effects on contractile function and myocardial metabolism [Cave 2000; Svedjeholm 1995]. Nevertheless, the role of GIK solution in cardiac surgery has a long and controversial history since the first introduction in 1962 in the setting of ischemic heart diseases [Cole 2015]. Over the past decades, a number of clinical studies reported that GIK could improve postoperative cardiac function, reduce need for inotropic support, lower the incidence of AF and shorten time ICU and hospital, whereas others failed to report any benefit [Koskenkari 2005; Lazar 2004; Smith 2002; Visser 2005]. In a previous meta-analysis, it showed there is no difference in the incidence of AF and mortality with the use of GIK in cardiac surgery [Ali-Hassan-Sayegh 2015; Rabi 2010]. So, there still is uncertainty regarding the efficacy and safety of GIK infusion in improving more clinically relevant end points. Recently, some new clinical trials suggested that the administration of GIK has a beneficial role in myocardial protection and resulted in better post-operative outcomes in patients undergoing on-pump cardiac surgery [Ahmad 2017; Ellenberger 2018; Licker 2019], but these have not, so far, been comprehensively and systematically reviewed.

Therefore, the purpose of this study is to perform a metaanalysis of the available evidence reporting the efficacy and safety of GIK infusion on clinical outcomes in adult patients undergoing on-pump cardiac surgery.

\section{METHOD}

Literature search strategy: All published randomized controlled trials (RCTs) that compared GIK administration with placebo or standard care in patients undergoing on-pump cardiac surgery were eligible for inclusion. The 


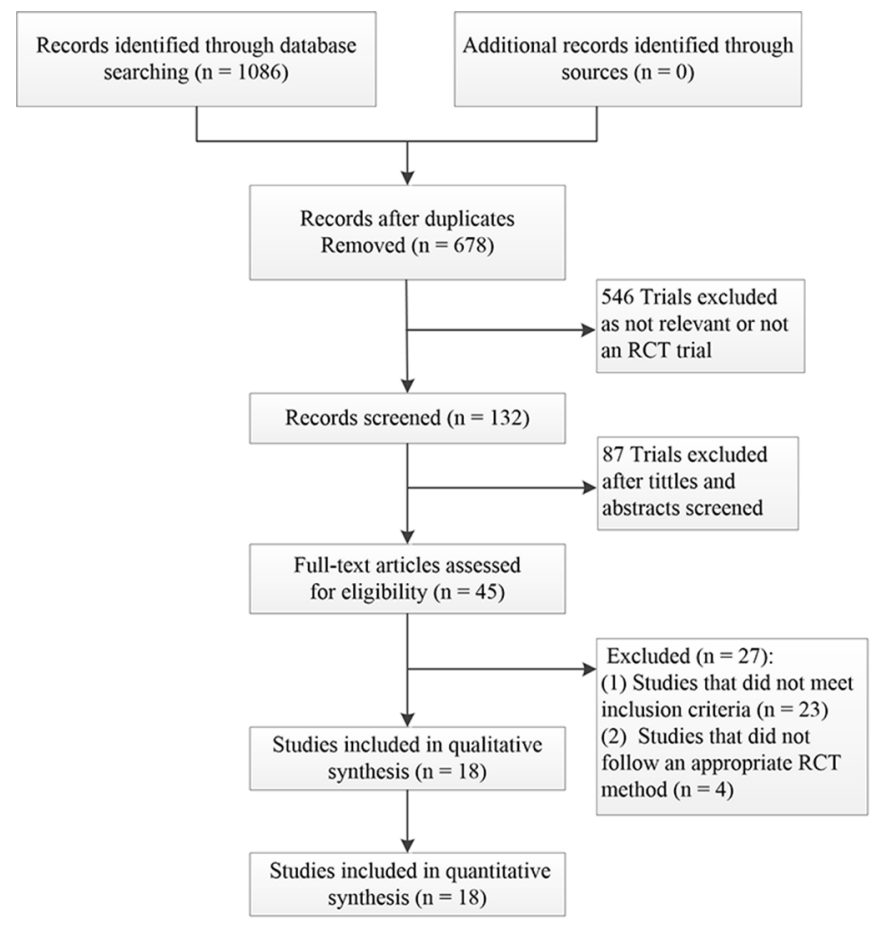

Figure 1. Flowchart illustrating the steps of the systematic review and meta-analysis.

PubMed, Medline, Web of Science, Embase, and Cochrane Library databases were searched by two independent reviewers, using the following keywords or search terms: glucoseinsulin-potassium, GIK, polarizing solution, cardiac surgery, heart surgery, on-pump, cardiopulmonary bypass, CPB, and randomized controlled trial. All eligible articles written in English were published between June 2000 and June 2019. Diverse combinations of free terms also were used, and various search strategies were developed for each database.

Study selection: Inclusion criteria were as follows: (1) RCTs; (2) all adult patients undergoing cardiac surgery requiring CPB; (3) comparison of GIK infusion versus placebo, or standard care; and (4) reporting data at least one of the primary and secondary outcomes. The primary end points were in-hospital mortality, postoperative $\mathrm{AF}$, and postoperative MI; the secondary outcomes of interest were infections, length of ICU stay, and postoperative use of inotropic support. Exclusion criteria were as follows: (1) non-human study, comments, reviews, letters, and case reports; (2) studies not reporting any of the outcomes mentioned above; (3) the data of studies had been duplicated in other publications; and (4) participants underwent transplantation or off-pump cardiac surgery.

Data extraction and quality assessment: The data from eligible articles were independently and separately abstracted by two reviewers, and discrepancies that arose during this process were resolved by consensus among co-authors. The potential moderator variables included the following: study design, patient numbers and characteristics, GIK protocols, type of surgical procedure, timing of solution infusion, and infusion methods.

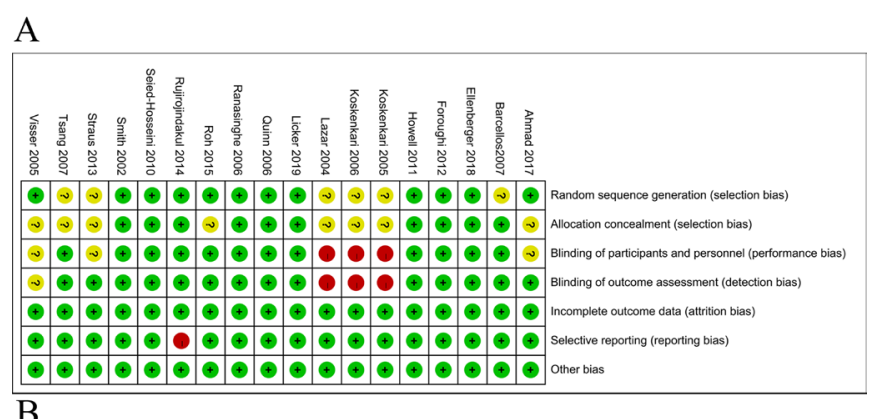

B

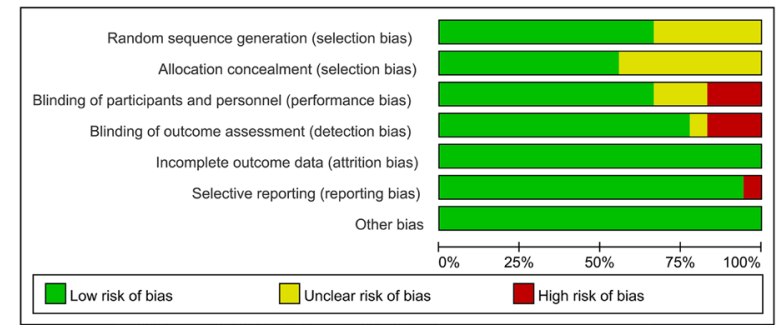

Figure 2. Evaluation of risk of bias for included studies: $(A)$, the result of bias risk; (B), the summary of risk bias.

After retrieving the full articles, the quality of included trials was evaluated according to the standards of the Cochrane Collaboration [Higgins 2011]. The assessment included the following: (1) random sequence generation; (2) concealment of allocation; (3) blinding of participants and personnel; (4) blinding of outcome assessment; (5) incomplete outcome data; (6) selective reporting; and (7) any other risk of bias. In each domain, studies were labeled as high, unclear, or low risk of bias with consideration given to the presence or absence of sufficient information to make a determination.

Statistical analysis: All statistical analyses were performed using Review Manager (version 5.3). Risk ratios (RR) were used for binary outcomes (i.e. death, MI, AF, infections, inotropic support) and mean difference (MD) was used for continuous variables (length of ICU stay); both with their $95 \%$ confidence intervals (CI). The statistical heterogeneity was assessed using the chi-squared and $\mathrm{I}^{2}$ tests. The fixed-effect model was preferentially reported in the absence of significant heterogeneity $\left(P>.1, \mathrm{I}^{2}<50 \%\right)$, whereas if heterogeneity was significant $\left(P<.1, \mathrm{I}^{2}>50 \%\right)$, a random-effect model analysis was performed. Additionally, publication bias was measured by using funnel plots. All the statistical significance was set at 0.05 .

\section{RESULTS}

Identification of eligible studies: A total of 1,086 citations were identified during the comprehensive literature search of all major databases. Of these citations, 633 studies were excluded based on titles and abstract review, and 27 studies were excluded after full-text review. Ultimately, 18 RCTs enrolling 2,131 participants met the inclusion criteria and were used for our meta-analysis [Ahmad 2017; 
A

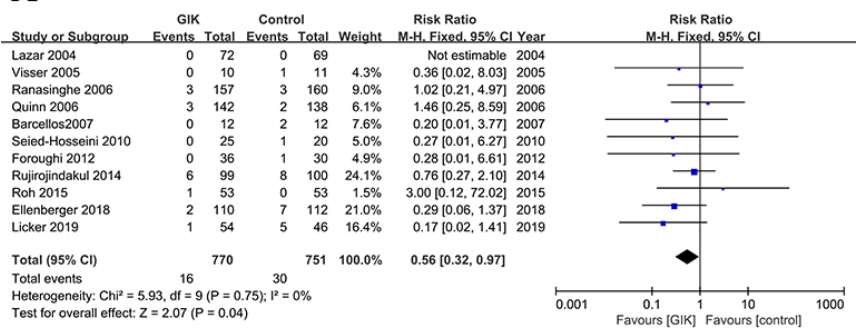

B

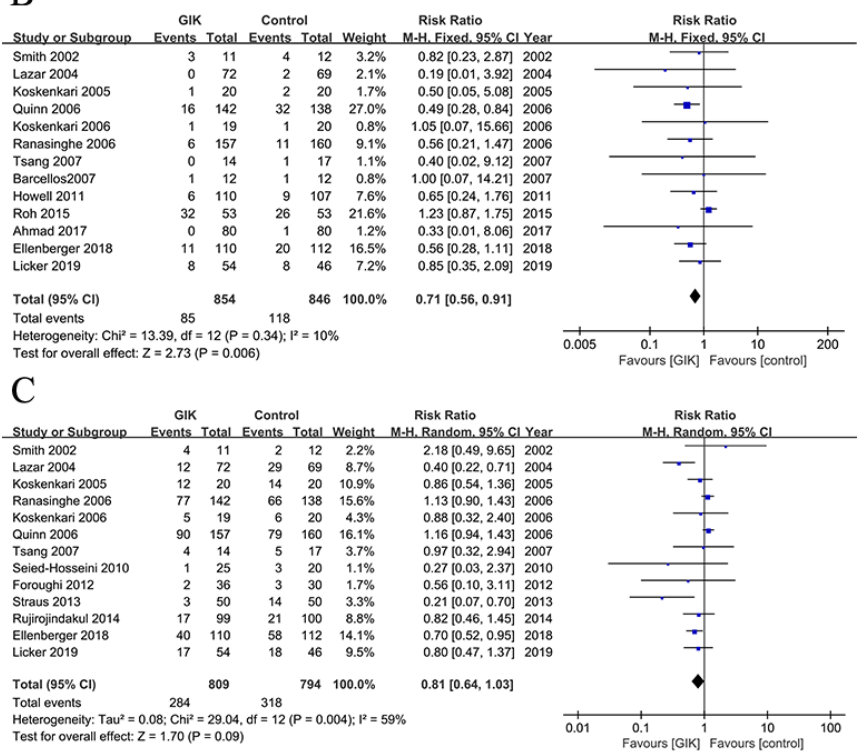

Figure 3. Forest plots of GIK therapy on the primary end points: (A), In-hospital mortality; (B), postoperative atrial fibrillation; (C), postoperative myocardial infarctions. GIK: Glucose-insulin-potassium, AF: atrial fibrillation, Ml: myocardial infarctions, Cl: confidence intervals. For authors' names and the years, they indicate the corresponding item in the "References."

Barcellos 2007; Ellenberger 2018; Foroughi 2012; Howell 2011; Koskenkari 2005; Koskenkari 2006; Lazar 2004; Licker 2019; Quinn 2006; Ranasinghe 2006; Roh 2015; Rujirojindakul 2014; Seied-Hosseini 2010; Smith 2002; Straus 2013; Tsang 2007; Visser 2005]. Figure 1 shows the details of the study selection process in the meta-analysis (Figure 1).

Characteristics of eligible studies: The characteristics of eligible RCTs are presented in the Table, including age, patient number, surgery type, and GIK intervention. The meta-analysis involved 2,131 total patients: 1,074 received GIK infusion around the time of cardiac surgery, and 1,057 received placebo or standard care (control group). All studies stated that patients were undergoing cardiac surgery requiring CPB. The majority of trials were conducted in the setting of coronary artery bypass grafting (CABG) surgery, 6 trials were performed using isolated valve or combined $\mathrm{CABG} /$ valve surgery.

Risk of bias in eligible studies: The risk of bias of the included studies are presented in Figure $2 \mathrm{~A}$ and the
A

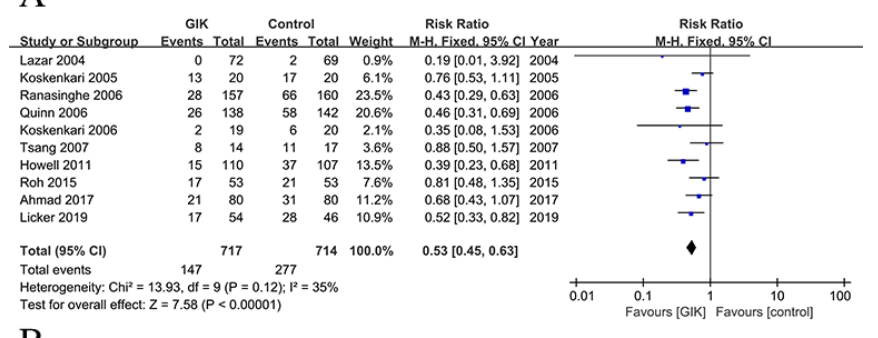

B

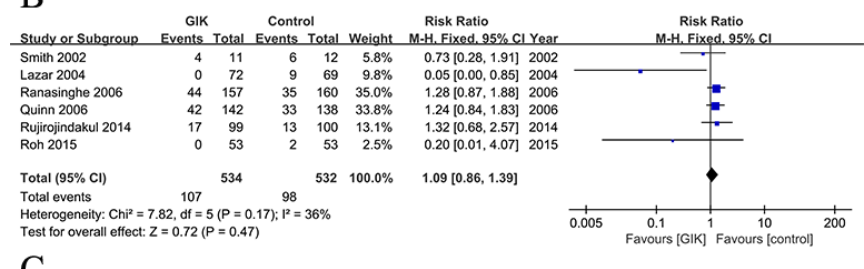

$\mathrm{C}$

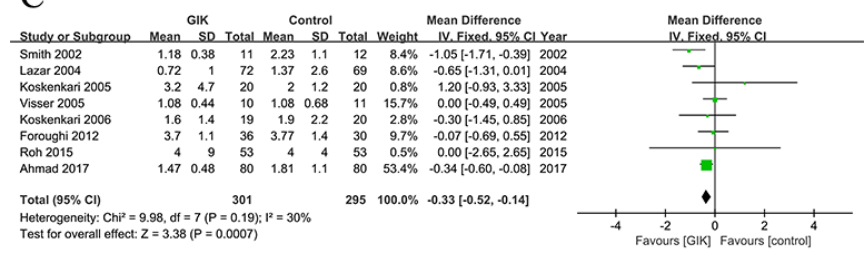

Figure 4. Forest plots of GIK therapy on the secondary end points: (A), Inotropic support; (B), Infection; (C), ICU length of stay. GIK: Glucoseinsulin-potassium, ICU: intensive care unit, $\mathrm{Cl}$ : confidence intervals. For authors' names and the years, they indicate the corresponding item in the "References."

percentage of studies for which we judged each item evaluated at as high, low, or unclear risk of bias are summarized in Figure 2B. Overall, the study quality was stable, with low risk of bias accounted for $>75 \%$ in most of the included studies. Based on a detailed evaluation of each studies, we observed that 12 studies could be considered as high quality because no high risk of bias was found. Three studies were classified as moderate quality due to unknown risk based on identification of potential selection bias and performance bias. The remaining 3 studies exhibited low quality because of multiple "high risk of bias" owing to lack of randomization and blind method (Figure 2).

Postoperative MI: Thirteen studies including 1,700 patients presented data for postoperative MI. The incidence rate of postoperative MI was $10.0 \%$ ( 85 out of 854 ) in the GIK group and $13.9 \%$ (118 out of 846 ) in the control group. GIK treatment was associated with decreased the incidence of postoperative $\mathrm{MI}(\mathrm{RR}=0.71,95 \% \mathrm{CI}: 0.56-0.91 ; P=$ .006 ) in the fixed model (Figure 3B). There was no significant heterogeneity seen $\left(P=.34, \mathrm{I}^{2}=10 \%\right)$.

Postoperative AF: Thirteen studies including 1,603 patients reported outcomes regarding postoperative AF. Postoperative $\mathrm{AF}$ occurred in $35.1 \%$ of patients (284 out of 809 ) in the GIK group, compared with of patients $40.1 \%$ of patients (318 out of 794) in the control group. There was a trend toward reduction in postoperative AF by GIK treatment $(\mathrm{RR}=0.81,95 \%$ CI: $0.64-1.03 ; P=.09$ ) in the random-effect model (Figure 3C). A moderate level of statistical heterogeneity was found 


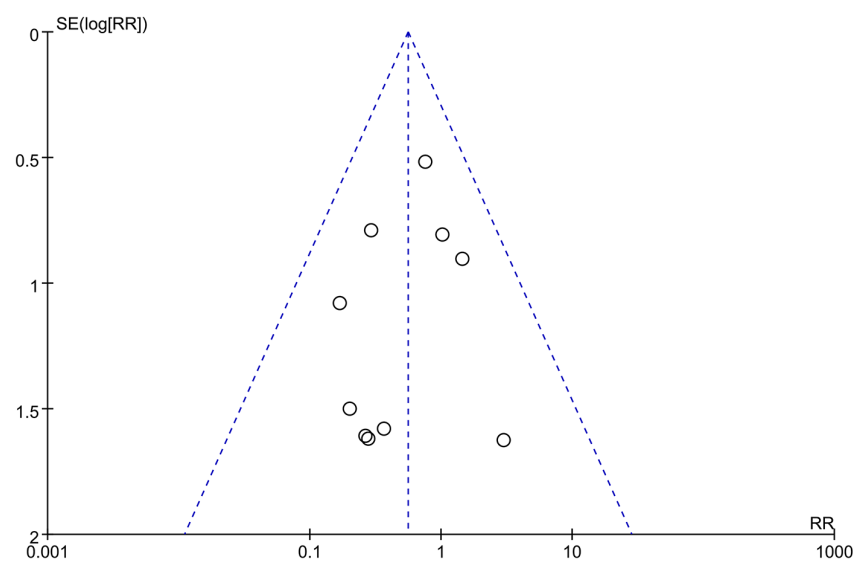

Figure 5. The funnel plot of the incidence of In-hospital mortality.

among the RCTs $\left(P=.004, \mathrm{I}^{2}=59 \%\right)$. Hence, we conducted a sensitivity analysis by excluding the two studies [Ranasinghe 2006; Quinn 2006] and repeated the analysis for this endpoint on remaining studies, yielding a pooled RR of 0.67 (95\% CI: $0.55-0.81 ; P<.0001)$, with a reduction in heterogeneity $(P=$ $.24, \mathrm{I}^{2}=21 \%$ ) (Figure 3).

Inotropic support: The inotropic support data was reported in 10 studies including 1,431 patients. The requirement for inotropic support was $20.5 \%$ (147 out of 717 ) in the GIK group and $38.8 \%$ (277 out of 714) in the control group. There was a significant reduction in the use of inotropic support for patients receiving GIK infusion (RR = $0.53,95 \%$ CI: $0.45-0.63 ; P<.00001)$ in the fixed model (Figure 4A). The heterogeneity was low in the analysis $(P=$ $\left..12, \mathrm{I}^{2}=35 \%\right)$.

Infection: 1,066 patients from 6 studies reported outcomes regarding infection after cardiac surgery. Among the RCTs, 107 of 534 patients $(20.0 \%)$ in the GIK group developed infection compared with 98 of 532 patients $(18.4 \%)$ in the control group. There was no significant difference between both groups, regarding the incidence of postoperative infection, with RR of 1.09 (95\% CI: 0.86 $-1.39 ; P=.47)$ in the fixed model (Figure $4 \mathrm{~B})$. Low statistical heterogeneity was observed in the analysis $(P=.17$, $\left.\mathrm{I}^{2}=36 \%\right)$.

ICU length of stay: 1,647 patients from 13 studies presented data for the ICU length of stay after cardiac surgery. Following the exclusion of 5 RCTs with incomplete data, 596 patients were analyzed. The mean length of ICU stays was 2.12 days in the GIK group and 2.28 days in the control group. There was a significant reduction in the length of ICU stay with patients receiving GIK treatment $(\mathrm{MD}=-0.33,95 \%$ CI: $-0.52--0.14 ; P=.0007)$ in the fixed model (Figure 4C). No significant heterogeneity was observed $\left(P=.19, \mathrm{I}^{2}=30 \%\right)$ (Figure 4).

Publication bias: Publication bias was visualized through funnel plots. The funnel plots for all causes of mortality in hospital showed that all points basically were symmetrical distribution, suggesting that no significant publication bias was found (Figure 5).

\section{DISCUSSION}

With the popularity of GIK infusion in patients undergoing cardiac surgery, a growing number of effects for this drug have been found. However, there still are many controversies that need to be resolved. Our meta-analysis of 18 RCTs involving more than 2,131 patients assess the safety and efficacy of GIK treatment in patients undergoing cardiac surgery requiring $\mathrm{CPB}$, demonstrating that the perioperative use of GIK infusions is associated with reduction in in-hospital mortality, postoperative MI, AF, the use of inotropic support and the length of ICU stay, as compared with a control group. All the included studies are based on a design of randomized, multicenter, double-blind trials, which relatively offered us concordant models.

It is well known that GIK provides glucose and insulin to stimulate myocardial glucose uptake and utilization. Augmentation of glucose utilization may be beneficial to myocardium because it needs a lower rate of oxygen consumption compared with fatty acid oxidation, thus improving cardiac efficiency and systolic function [Stanley 2002]. On the other hand, GIK infusion may play a role in cardioprotective effects in cardiac surgery owing to reducing free fatty acid oxidation, promoting glucose as the primary myocardial energy substrate, stabilizing myocardial membrane electrical gradient, and enhancing cell survival while decreasing hyper/hypoglycemic events [ $\mathrm{Ng}$ 2012]. Moreover, GIK also has a role in insulin signaled K-ATP channels activation. These channels are a vital regulator of ischemic preconditioning that have strong protective effects on $\mathrm{MI}$ and ischemia reperfusion injury [Ichinomiya 2012; Prendes 2014]. In a preclinical large animal model, treatment with GIK exerted obviously cardioprotective effects as evidenced by improved cardiac function and coronary blood flow, reduced myocardial apoptosis and infarct size [Zhang 2006]. Several experimental studies reported that GIK infusion improves regional left ventricular function and allows the detection of myocardial viability to a similar extent as low-dose dobutamine in patients shortly after infarction [Klein 2006]. These important potential benefits in cardiovascular diseases and cardiac surgery explain why GIK has held interest for so many years.

Interestingly, contrary to our positive findings of GIK treatment in patients undergoing cardiac surgery, the large, international randomized trial (CREATE-ECLA trial) of GIK treatment for ST-segment elevation myocardial infarction (STEMI) demonstrates that high-dose GIK infusion had a neutral effect on mortality, cardiogenic shock, and cardiac arrest in patients with acute STEMI [Mehta 2005]. Besides a recent meta-analysis of $25 \mathrm{RCTs}$ has reported that, in patients undergoing GABG, GIK did not have considerable cardioprotective effects including the incidence of postoperative AF, MI, and infection [Ali-Hassan-Sayegh 2015]. Another metaanalysis of 20 RCTs also has concluded that the perioperative use of GIK or GIK without potassium does not significantly reduce mortality or AF in patients undergoing CABG surgery [Rabi 2010]. There are some potential explanations for this conflicting evidence, and the main reason may be the fundamental differences in GIK dose, timing, composition, or 
Characteristics of the Clinical Trials Included in the Meta-analysis *

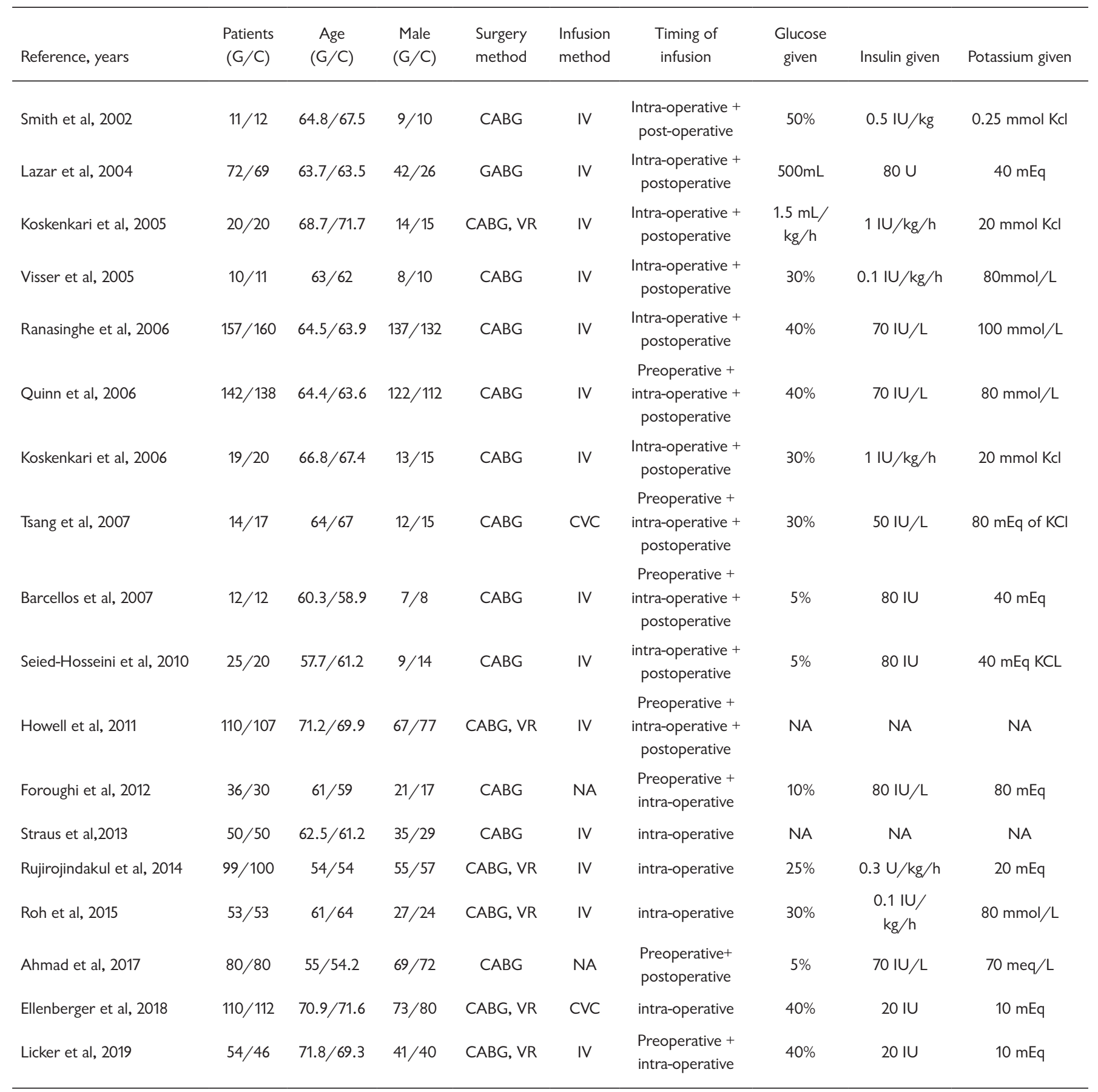

*GIK, Glucose-insulin-potassium; NA, not available; CABG, Coronary Artery Bypass Graft; VR, Valve Replacement, include Aortic Valve Replacement and Mitral Valve Replacement; IV, intravenously; CVC, central venous catheter.

duration of administration. Another important reason might be that the adverse effects of hyperglycemia may offset the benefits from GIK infusion [LaDisa 2004].

Our meta-analysis includes the latest RCTs in the past 20 years. Compared with early clinical studies, most of RCTs were double blinded and high quality, decreasing performance and detection bias and the statistical analysis showed significant difference. Based on our findings, it can be concluded that GIK infusion was beneficial to reduce postoperative MI, the risk of death, use of inotropic support as well as length 
of ICU stay in patients undergoing on-pump cardiac surgery. Of note, there is moderate level of statistical heterogeneity in the results of postoperative AF. Further analysis indicates that the Ranasinghe [Ranasinghe 2006] and Quinn [Quinn 2006] studies might have contributed to heterogeneity existed. The explanation might be that the dose of insulin and potassium in the 2 studies is higher than other studies, and the incidence of complications, such as hyperkalemia and hypoglycemia, might increase what may offset the benefits from GIK infusion. Thus, blood glucose and potassium levels must frequently be monitored. Although there was a significant reduction in the incidence of $\mathrm{AF}$ as well as with a reduction in heterogeneity by excluding the 2 studies, this outcome still needs to cautiously be interpreted. Additionally, we found that GIK therapy is not able to decrease the incidence of postoperative infection and this finding is consistent with the prior meta-analysis [AliHassan-Sayegh 2015]. Further studies are required to demonstrate the role of GIK in anti-inflammatory and postoperative infection during cardiac surgery.

\section{STUDY LIMITATIONS}

There are several limitations to consider when interpreting the results of our meta-analysis. First, many end points such as $\mathrm{AF}, \mathrm{MI}$ and mortality were not the primary outcomes in many of the RCTs included in the present study. These RCTs were, in large part, examining the metabolic and physiological consequences of the GIK treatment during cardiac surgery. Thus, it is possible that the tests had inadequate power. Second, several potentially confounding factors exist in the included RCTs, including inclusion of diabetic patients, type of surgical procedure, ingredients of GIK therapy, and timing of the solution infusion, which would affect the outcome of the analysis. Third, although our study found that GIK treatment could significantly reduce the incidence of postoperative MI, AF, risk of death and use of inotropic support, further investigation of the effect of GIK on cardiac index, renal disease and stroke after cardiac surgery is necessary to provide better understanding of the role of GIK infusion. Finally, because of the low event rates and the lack of follow-up, we were unable to access the long-term effects of GIK infusion.

\section{CONCLUSION}

Despite the potential limitations, this meta-analysis represents strong statistical evidence to support the perioperative use of GIK to decrease the incidence of postoperative MI, $\mathrm{AF}$, and in-hospital mortality in patients undergoing cardiac surgery requiring CPB. Furthermore, GIK infusion is associated with less requirement for inotropic support and a shorter length of stay in ICU. This may provide a safe efficacy therapy for patients undergoing cardiac surgery requiring $\mathrm{CPB}$. Nevertheless, there remains room for refinement of therapy identification between the total insulin dosage and mean glucose concentration, which could derive greatest benefit.
REFERENCES

Ahmad S, Ahmad RA, Qureshi BA, Baig M. 2017. Myocardial protection with Glucose-Insulin-Potassium infusion during adult cardiac surgery. Pak J Med Sci 33:325-329.

Ali-Hassan-Sayegh S, Mirhosseini SJ, Zeriouh M, et al. 2015. Safety and efficacy of glucose-insulin-potassium treatment in coronary artery bypass graft surgery and percutaneous coronary intervention. Interact Cardiovasc Thorac Surg 21:667-76.

Barcellos CS, Wender OC, Azambuja PC. 2007. Clinical and hemodynamic outcome following coronary artery bypass surgery in diabetic patients using glucose-insulin-potassium (GIK) solution: A randomized clinical trial. Rev Bras Cir Cardiovasc 22:275-84.

Benjamin EJ, Virani SS, Callaway CW, et al. 2018. Heart disease and stroke statistics-2018 update: A report from the american heart association. Circulation 137:e67-e492.

Cave AC, Ingwall JS, Friedrich J, et al. 2000. ATP synthesis during lowflow ischemia: Influence of increased glycolytic substrate. Circulation 101:2090-6.

Cole SP, Gross ER. 2015. Happy 53rd Birthday GIK: insulin, cake, and presents. Anesthesiology 123:249-50.

Ellenberger C, Sologashvili T, Kreienbuhl L, et al. 2018. Myocardial protection by Glucose-Insulin-Potassium in moderate- to High-Risk patients undergoing elective On-Pump cardiac surgery: A randomized controlled trial. Anesthesiology 126:1133-1141.

Foroughi M, Rahimian H, Dabbagh A, et al. 2012. Postoperative N-terminal pro-brain natriuretic peptide level in coronary artery bypass surgery with ventricular dysfunction after perioperative glucose-insulinpotassium treatment. J Cardiothorac Vasc Anesth 26:631-6.

Giomarelli P, Scolletta S, Borrelli E, Biagioli B. 2003. Myocardial and lung injury after cardiopulmonary bypass: Role of interleukin (IL)-10. Ann Thorac Surg 76:117-23.

Higgins JP, Altman DG, Gotzsche PC, et al. 2011. The Cochrane Collaboration's tool for assessing risk of bias in randomised trials. BMJ 343:d5928.

Howell NJ, Ashrafian H, Drury NE, et al. 2011. Glucose-insulin-potassium reduces the incidence of low cardiac output episodes after aortic valve replacement for aortic stenosis in patients with left ventricular hypertrophy: Results from the Hypertrophy, Insulin, Glucose, and Electrolytes (HINGE) trial. Circulation 123:170-7.

Ichinomiya T, Cho S, Higashijima U, et al. 2012. High-dose fasudil preserves postconditioning against myocardial infarction under hyperglycemia in rats: Role of mitochondrial KATP channels. Cardiovasc Diabetol 11:28.

Klein LJ, van Campen LC, Sieswerda GT, et al. 2006. Glucose-insulinpotassium echocardiography detects improved segmental myocardial function and viable tissue shortly after acute myocardial infarction. J Am Soc Echocardiogr 19:763-71.

Koskenkari JK, Kaukoranta PK, Kiviluoma KT, et al. 2005. Metabolic and hemodynamic effects of high-dose insulin treatment in aortic valve and coronary surgery. Ann Thorac Surg 80:511-7.

Koskenkari JK, Kaukoranta PK, Rimpilainen J, et al. 2006. Anti-inflammatory effect of high-dose insulin treatment after urgent coronary revascularization surgery. Acta Anaesthesiol Scand 50:962-9.

LaDisa JJ, Krolikowski JG, Pagel PS, Warltier DC, Kersten JR. 2004. Cardioprotection by glucose-insulin-potassium: Dependence on KATP channel opening and blood glucose concentration before ischemia. Am J 
Physiol Heart Circ Physiol 287:H601-7.

Laslett LJ, Alagona PJ, Clark BR, et al. 2012. The worldwide environment of cardiovascular disease: Prevalence, diagnosis, therapy, and policy issues: A report from the American College of Cardiology. J Am Coll Cardiol 60:S1-49.

Lazar HL, Chipkin SR, Fitzgerald CA, et al. 2004. Tight glycemic control in diabetic coronary artery bypass graft patients improves perioperative outcomes and decreases recurrent ischemic events. Circulation 109:1497-502.

Licker M, Reynaud T, Garofano N, et al. 2019. Pretreatment with glucose-insulin-potassium improves ventricular performances after coronary artery bypass surgery: A randomized controlled trial. J Clin Monit Comput.

Mathew JP, Fontes ML, Tudor IC, et al. 2004. A multicenter risk index for atrial fibrillation after cardiac surgery. JAMA 291:1720-9.

Mehta SR, Yusuf S, Diaz R, et al. 2005. Effect of glucose-insulin-potassium infusion on mortality in patients with acute ST-segment elevation myocardial infarction: The CREATE-ECLA randomized controlled trial. JAMA 293:437-46.

Ng KW, Allen ML, Desai A, Macrae D, Pathan N. 2012. Cardioprotective effects of insulin: How intensive insulin therapy may benefit cardiac surgery patients. Circulation 125:721-8.

Prendes MG, Hermann R, Torresin ME, et al. 2014. Role of mitochondrial permeability transition pore and mitochondrial ATP-sensitive potassium channels in the protective effects of ischemic preconditioning in isolated hearts from fed and fasted rats. J Physiol Biochem 70:791-800.

Quinn DW, Pagano D, Bonser RS, et al. 2006. Improved myocardial protection during coronary artery surgery with glucose-insulin-potassium: A randomized controlled trial. J Thorac Cardiovasc Surg 131:34-42.

Rabi D, Clement F, McAlister F, et al. 2010. Effect of perioperative glucose-insulin-potassium infusions on mortality and atrial fibrillation after coronary artery bypass grafting: A systematic review and meta-analysis. Can J Cardiol 26:178-84.

Ranasinghe AM, Quinn DW, Pagano D, et al. 2006. Glucose-insulinpotassium and tri-iodothyronine individually improve hemodynamic performance and are associated with reduced troponin I release after on-pump coronary artery bypass grafting. Circulation 114:I245-50.

Roh GU, Shim JK, Song JW, Kang HM, Kwak YL. 2015. Effect of glucose-insulin-potassium on hyperlactataemia in patients undergoing valvular heart surgery: A randomised controlled study. Eur J Anaesthesiol $32: 555-62$.

Rujirojindakul P, Liabsuetrakul T, McNeil E, et al. 2014. Safety and efficacy of intensive intraoperative glycaemic control in cardiopulmonary bypass surgery: A randomised trial. Acta Anaesthesiol Scand 58:588-96.

Seied-Hosseini SM, Pourmoghadas A, Aghadavoudi O, et al. 2010. Efficacy of Glucose-Insulin-Potassium infusion on left ventricular performance in type II diabetic patients undergoing elective coronary artery bypass graft.dy. ARYA Atheroscler 6:62-8.

Smith A, Grattan A, Harper M, Royston D, Riedel BJ. 2002. Coronary revascularization: A procedure in transition from on-pump to off-pump? The role of glucose-insulin-potassium revisited in a randomized, placebo-controlled study. J Cardiothorac Vasc Anesth 16:413-20.

Stanley WC, Chandler MP. 2002. Energy metabolism in the normal and failing heart: Potential for therapeutic interventions. Hear Fail Rev 7:115-30.

Straus S, Gerc V, Kacila M, Faruk C. 2013. Glucosa-Insulin-Potassium (GIK) solution used with diabetic patients provides better recovery after coronary bypass operations. Med Arch 67:84-7.

Svedjeholm R, Huljebrant I, Hakanson E, Vanhanen I. 1995. Glutamate and high-dose glucose-insulin-potassium (GIK) in the treatment of severe cardiac failure after cardiac operations. Ann Thorac Surg 59:S23-30.

Tsang MW, Davidoff R, Korach A, et al. 2007. Diastolic dysfunction after coronary artery bypass grafting--the effect of glucose-insulin-potassium infusion. J Card Surg 22:185-91.

Visser L, Zuurbier CJ, Hoek FJ, et al. 2005. Glucose, insulin and potassium applied as perioperative hyperinsulinaemic normoglycaemic clamp: Effects on inflammatory response during coronary artery surgery. Br J Anaesth 95:448-57.

Zhang HX, Zang YM, Huo JH, et al. 2006. Physiologically tolerable insulin reduces myocardial injury and improves cardiac functional recovery in myocardial ischemic/reperfused dogs. J Cardiovasc Pharmacol 48:306-13. 